Trauma Berufskrankh 2006 • 8[Suppl 3]:S299-S302 DOI 10.1007/s10039-005-1074-y

๑) Springer Medizin Verlag 2006
Online publiziert: 5 . Januar 2006

\author{
J.W. Weidringer ${ }^{1}$ P. Sefrin ${ }^{2}$ \\ ${ }^{1}$ Bayerische Landesärztekammer, München \\ ${ }^{2}$ Sektion präklinische Notfallmedizin, Klinik und Poliklinik für Anästhesiologie, \\ Zentrum Operative Medizin, Universität Würzburg
}

\section{Vorteile der Laienreanimation}

wende nicht nur eine Vielzahl von AED („automatic external defibrillators") installiert, sondern auch dort Beschäftigte gemeinsam mit der regional ansässigen Bevölkerung in einer beispielhaft intensiven Aufklärungskampagne zu Basiswiederbelebungsmaßnahmen sowie dem Einsatz von AED informiert. Die 2002 von Caffrey et al. [10] veröffentlichte Chicago-Studie zeigte die Ergebnisse dieser Maßnahme: In der Studie enthalten sind 26 Patienten, 22 dieser 26 erlitten einen Herzstillstand, bei 21 mit nichttraumatischer Genese, 1 Patient verstarb sofort. Bei 20 der 22 Patienten handelte es sich um einen beobachteten Herzstillstand, 2 dieser Patienten zeigten elektrische Restaktivität, 18 Kammerflimmern (2 Frauen, 16 Männer). Bei allen erfolgte die AED-Anwendung durch Laien. 7 Patienten verstarben, wobei es sicherlich eine Rolle spielte, dass die AED z. T. erst nach 5 min verfügbar war, 11 Patienten wurden mit gutem neurologischem Befund entlassen, bei 10 Patienten wurde eine Überlebenszeit von länger als $1 \mathrm{Jahr}$ erreicht.
Die initial installierten 67 AED haben in einem Zeitraum von 10 Jahren Kosten von 35.000 US\$/Jahr verursacht.

\section{Internationales Prozedere}

In den USA wurden ab der 2. Hälfte der 9oer Jahre primär in den verschiedensten öffentlichen Bereichen AED verfügbar gemacht. Beispielsweise sind an den Flughäfen Newark, New Jersey sowie am San Francisco International Airport in einer Entfernung von nur wenigen 100 Yards teils mehrere AED öffentlich zugänglich montiert (!).

Auch in Europa sind in vielen Großstädten sowie an Flughäfen AED öffentlich zugänglich - dabei unterscheiden sich das "Verteilungs“- sowie „Zugänglichkeits“Muster in verschiedenen deutschen Großstädten teils nachhaltig, was je nach regionaler rettungsdienstlicher Einsatzstruktur z. T. nachvollziehbar scheint.

Bei näherer Beschäftigung mit der Thematik stellt sich in vielen Fällen heraus, erkennen lassen, dass zum einen innerhalb der ersten 10 Minuten nach Kammerflimmern je Minute die Überlebenswahrscheinlichkeit um etwa $7 \%$ abnimmt, zum anderen nur eine sehr frühe, eben auch für Laien mögliche, automatisierte Defibrillation während der ersten 4-5 min, wohlgemerkt im Kontext mit kardiopulmonaler Reanimation, eine etwa 50\%ige Überlebenschance bringt (• Abb. 1, 2).

\section{AED (,, automatic external defibrillators")}

\section{Chicago}

Am International Airport O'Hare, Chicago, Illinois, sowie in dessen unmittelbarer Umgebung wurden um die Jahrtausend-

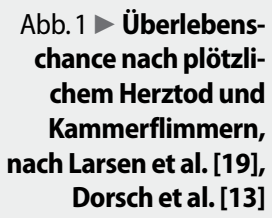




\section{ATLS und Laienreanimation}

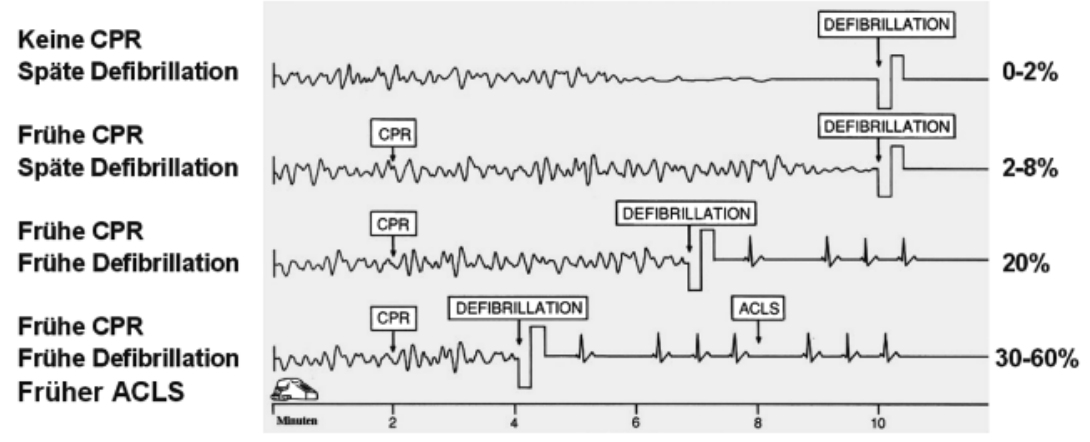

Abb. $2<$ Überlebensraten in Abhängigkeit vom Zeitpunkt der kardiopulmonalen Reanimation, Defibrillation sowie erweiterten Reanimationsmaßnahmen, nach American Heart Association [1, 2, 3]

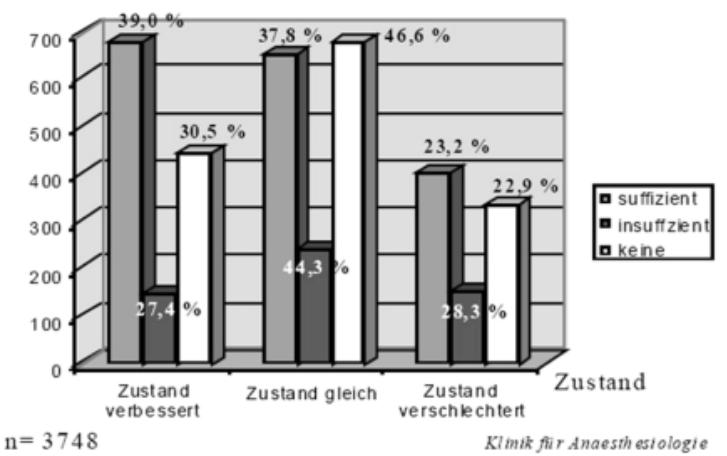

Abb. $3 \Delta$ Ersthelfermaßnahmen bei Reanimation, Zustand bei Patientenübergabe, Auswertung von 200.221 Notarzteinsätzen in Bayern

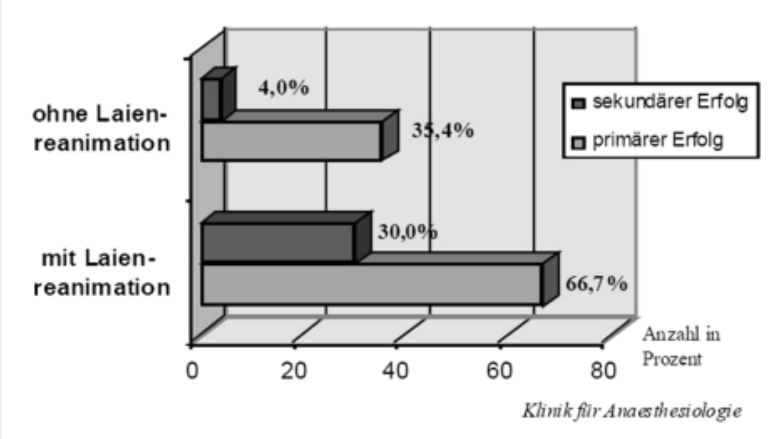

Abb. $4 \Delta$ Reanimationserfolg im Göttinger Laienreanimationsprogramm dass die dokumentierten und öffentlich publik gemachten AED-Anwendungen die für die Betroffenen im wahrsten Sinne des Wortes lebensrettend waren - in einer Vielzahl von Fällen keineswegs von Laien, sondern von letztlich qualifizierten Ersthelfern realisiert wurden.

In Fachkreisen nicht unerwartet haben die Studienergebnisse von Zipes [37] und Zipes u. Wellens [38] insofern (relatives) Aufsehen erregt, als klar herausgearbeitet wurde, dass plötzliche Herz-KreislaufStillstände sich seltener im öffentlichen Bereich, vielmehr eher - in $>70 \%$ aller Fälle - im häuslichen Umfeld ereignen. Es sei an dieser Stelle erwähnt, dass mittlerweile Kampagnen erkennbar sind, die die Ausstattung von beispielsweise Senioren- und Altenpflegeheimen mit AED fordern ...

\section{Laien-Herz-Lungen-Wieder- belebung sowie (öffentlich zugängliche) Frühdefibrillation}

Eine Studie der Sektion Präklinische Notfallmedizin des Universitätsklinikums Würzburg $[30,32]$ kam ebenso wie eine unabhängig davon durchgeführte Untersuchung im Rahmen des so genannten „Göttinger Laienreanimationsprogramms" [11] zu dem Schluss, dass die Laienreanimation das überlebenswichtige Glied in der Rettungskette darstellt (• Abb. 3, 4). Beim Laienreanimationsprogramm in Göttingen [11] zeigte sich - ohne Laiendefibrillation - beim primären Erfolg eine Verdoppelung und beim sekundären Erfolg sogar eine 7,5fach höhere Quote des Überlebens.

\section{Qualifizierungs- und Trainingsmöglichkeiten}

Geradezu vorbildlich adressieren die Berufsgenossenschaften im „Handbuch zur Ersten Hilfe" (BGl 829, Grundsätze der Bundesarbeitsgemeinschaft Erste Hilfe vom 20.03.2003) in mehr als 3 Mio. Unternehmen indirekt über 43 Mio. Versicherte - mit jeweils hoch aktuellen und richtigen Handlungsanleitungen, wie beispielsweise... „bei fehlenden Lebenszeichen ohne Pulskontrolle unverzüglich die Herz-Lungen-Wiederbelebung durchzuführen“... Das obligate BG-Ausbildungskonzept „Erste Hilfe im Betrieb“ wird via http://www.bgqseh.de webbasiert unterstützt und begleitet. Die hier ebenfalls aufgeführten Hinweise der Bundesarbeitsgemeinschaft Erste Hilfe vom 20.06.2003 sind in das berufsgenossenschaftliche Konzept integriert (http://www.bg-qseh.de).

Für im Rahmen der Frühdefibrillationsprogramme tätig werdende Ärztinnen und Ärzte sind relevante Hinweise im Deutschen Ärzteblatt vom 22.12.2003 [5] sowie korrespondierend hierzu unter http://www.aerzteblatt.de/v4/archiv/artikel.asp?id=39922 abrufbar. Weitere webbasierte hilfreiche Hinweise zur BasisHerz-Lungen-Wiederbelebung sowie zur Anwendung von AED im Sinne von zielgruppenorientierten Lernprogrammen finden sich im Internet unter http://www. aed-bayern.de.

Höchst nachdenklich können die Publikationen von Swor u. Compton [34] von Ende 2004 sowie von Groeneveld u. Owens [16] aus dem Januar 2005 stimmen: In beiden Publikationen kamen die Autoren in ihrem Resümee letztendlich für das ameri- 
kanische Umfeld zu dem Schluss, dass die derzeitigen Strategien zur Herz-LungenWiederbelebung für im häuslichen Umfeld auftretende Herz-Kreislauf-Stillstände nicht rentable Maßnahmen sind, vielmehr relativ kostenträchtig im Vergleich zu anderen Maßnahmen der öffentlichen $\mathrm{Ge}$ sundheitsfürsorge. Allerdings beinhaltete die Studie von Groeneveld u. Owens [16] auch eine aus Sicht der Autoren gerade für den Verantwortungsbereich der Berufsgenossenschaft und dort Beschäftigter positive Perspektive: Das Training von Laien in Abhängigkeit von ihrer beruflichen Tätigkeit, niedrigen Trainingskosten oder dem Leben mit Hochrisikopersonen in häuslicher Gemeinschaft ist definitiv effizienter.

In der Studie von Trappe et al. [34] aus dem Jahr 2005 wurde die positive Korrelation von Trainingszeit sowie -intensität einerseits und dem Reanimationserfolg andererseits betont.

\section{Fazit}

1. Jede Minute bis zur indizierten (automatisierten) externen Defibrillation erniedrigt die individuelle tertiäre Überlebenschance innerhalb der ersten 10 min beim Kammerflimmern.

2. Über $70 \%$ plötzlicher Herzstillstände geschehen im häuslichen Umfeld.

3. Die AED-Kampagnen zum „Public Access/Öffentlicher Anwendbarkeit" fokussieren auf Gebäude mit Publikumsverkehr; öffentlichkeitswirksame Aufklärung zu nicht nur AED-, sondern v. a. BLS-Anwendung durch „Laien“ scheinen Erfolg zu zeigen.

4. Im Interesse klarer Verantwortlichkeiten und ggf. passender (Frühdefibrillations-)Trainingsprogramme dürfte eine eindeutige Zielgruppenbenennung bezüglich der AED-Anwendung wichtig sein:

- Laien - im Sinne von „Bürger auf der Straße" -

- Ersthelfer (16 h Qualifizierung usw.)

- Medizinisches Assistenzpersonal gemäß vorbestehenden Qualifikationen

\section{- "Nichtärzte"}

5. Studien in Deutschland zeigten - abstrakt betrachtet - den Überlebensnutzen von Laien-Herz-Lungen-Wiederbelebung.
Trauma Berufskrankh $2006 \cdot 8$ [Suppl 3]:S299-S302

DOI 10.1007/s10039-005-1074-y

(c) Springer Medizin Verlag 2006

J.W. Weidringer $\cdot$ P. Sefrin

\section{Vorteile der Laienreanimation}

\section{Zusammenfassung}

Die Erhöhung der Überlebenswahrscheinlichkeit nach Herzstillstand mit Kammerflimmern durch frühzeitige Defibrillation und effektive Herz-Lungen-Wiederbelebung stellt ein Optimum für die Patientenversorgung dar. In Deutschland gibt es zwar standardisierte Trainingskonzepte für „Ersthelfer" und medizinisches Assistenzpersonal, dennoch ist die Umsetzung der öffentlich zugänglichen Frühdefibrillation höchst unterschiedlich realisiert. Aus amerikanischen sowie deutschen Studien resultieren Knotenpunkte für ein vielleicht neu zu spannendes Netz überlebenswichtiger Maßnahmen: Die Caffrey-Studie zeigte eine regional hohe Effizienz der Anwendung von Frühdefibrillation und Herz-LungenWiederbelebung, in 2 deutschen Studien wurde die überlebenswichtige Bedeutung der Laienreanimation nachgewiesen, die Studien von Zipes (USA) belegten, dass der

Tod durch Kreislaufstillstand in über $70 \%$ der Fälle im häuslichen Umfeld eintritt, in 2 aktuellen amerikanischen Studien wurden die Effizienz breit angelegter Laienreanimationsprogramme bezweifelt und dezidiert tätigkeits-/risikogruppenbezogene Trainings für Laien hinsichtlich Frühdefibrillation und Herz-Lungen-Wiederbelebung favorisiert. Dies aufgreifend wird auf die besondere Rolle der Berufsgenossenschaften mit u. a. qualifizierten Erste-Hilfe-Trainingsprogrammen für mehr als 43 Mio. Beschäftigte in mehr als 3 Mio. Betrieben eingegangen. Für das notfallmedizinische Netz muss eine Synthese zwischen Sozioökonomie und Ethik gefunden werden.

\section{Schlüsselwörter}

Frühdefibrillation · Herz-Lungen-

Wiederbelebung · Laienreanimation .

Effizienz $\cdot$ Ethik

\section{Advantages of lay person reanimation}

\section{Abstract}

Early defibrillation along with well performed basic life support (BLS) after cardiac arrest, due to ventricular fibrillation is the gold standard for increasing patients survival. Within Germany there are two standardized schemes for the training of first responders as well as of paramedics, emergency medical technicians etc.; however, the use of public access defibrillation (PAD) is highly variable between localities. Results from the USA as well as from Germany indicate approaches to life-saving issues which need addressing. The paper by Caffrey reveals high regional efficiency for PAD along with BLS, while two German studies show the value of lay individuals in BLS. Zipes (USA) study shows that death due to cardiac arrest occurs at the patient's home in more than $70 \%$. Two recent, standardized US trials cast doubt on the efficacy of broadly deployed BLS-training programs for lay persons, preferring PAD and BLS training carried out strictly in accordance with the riskratio, for instance, of the people near to the first responders. Taking this into account, social insurance for occupational accidents offer qualified first responder training for more than 43 million employees (about half of the Germany population) in more than 3 million companies. It is necessary to find a synthesis between socio-economic demands on the one hand and ethical principles on the other for the emergency medicine network.

\section{Keywords}

Public access defibrillation .

Basic life support - Lay person BLS .

Efficacy/efficiency · Ethical codes 


\section{Perspektiven}

1. Intensive industrielle Marketing- und Vertriebsaktivitäten zu automatisierten externen Defibrillatoren (AED) wirken wasserfallartig und bringen Bewegung auch in die öffentliche Wahrnehmung von Grenzsituationen des menschlichen Lebens.

2. Erkennbar ist eine erste allgemeine Bewusstseinsbildung zur Basiswiederbelebungswirksamkeit - v. a. für das „, therapiearme", erste Intervall.

3. Die sozioökonomische und v. a. ethische Dimension einer Kosten-NutzenAnalyse der Laienreanimation hat in Europa erst begonnen - und wird uns alle fordern.

\section{Korrespondierender Autor Dr. J.W. Weidringer}

Bayerische Landesärztekammer, Mühlbaurstraße 16, 81677 München E-Mail: j.w.weidringer@blaek.de

Danksagung. Die Autoren danken Frau Billi Ryska für die bekannt sorgfältige Textverarbeitung.

Interessenkonflikt: Keine Angaben

\section{Literatur}

1. American Heart Association (1992) Guidelines for cardiopulmonary resuscitation and emergency cardiac care. Emergency Cardiac Care Committee and Subcommittees, American Heart Association. JAMA 268: 2199ff

2. American Heart Association (1994) Textbook of advanced cardiac life support. American Heart Association, Dallas

3. American Heart Association (2002) AHA guidelines for primary prevention of cardiovascular disease and stroke: 2002 Update. Circulation 106: 388

4. Arntz HR (1993) Kardiopulmonale Reanimation beim Erwachsenen. Dtsch Med Wochenschr 118: 1289-1291

5. Arzneimittelkommission der deutschen Ärzteschaft (2003) Aus der UAW-Datenbank. Dtsch Arztebl 51-52: 3407

6. Bahr S, Busse C, Kettler HD (1989) Herz-LungenWiederbelebung durch Ersthelfer. Nofallmedizin 15: 53-62

7. Bundesarbeitsgemeinschaft Erste Hilfe (2003) Gemeinsame Grundsätze zur Aus- und Fortbildung von Ersthelfern in Frühdefibrillation; Fassung vom 30.06.2003, Eigendruck im Selbstverlag. Bundesarbeitsgemeinschaft Erste Hilfe, Berlin

8. Bundesärztekammer (2001) Empfehlungen der Bundesärztekammer zur Defibrillation mit automatisierten externen Defibrillatoren (AED) durch Laien (2001). Dtsch Ärztebl 98: A-1211/B-0135/C-967
9. Bundesärztekammer (2003) Stellungnahme der Bundesärztekammer zur ärztlichen Verantwortung für die Aus- und Fortbildung von Nichtärzten in der Frühdefibrillation. Dtsch Ärztebl 100: A-3407

10. Caffrey SL, Willoughby PJ, Pepe PE et al. (2002) Public use of automated external defibrillators. $\mathrm{N}$ Engl J Med 347: 1242-1247

11. Callies A (2000) Laienreanimation: notwendige Ergänzung eines optimalen Rettungsdienstes. Rettungsdienst 23: 774-779

12. Capucci A, Aschieri D, Piepoli MF et al. (2002) Tripling survival from sudden cardiac arrest via early defibrillation without traditional education in cardiopulmonary resuscitation. Circulation 106: 1065-1070

13. Dorsch A (2000) Pers. Unterlagen. Circulation 102: 61

14. Eisenberg MS, Horwood BT, Cummins RO et al. (1990) Cardiac arrest and resuscitation: a tale of 29? Ann Emerg Med 19: 179-186

15. Fenster JS, Lopez M, Lai S et al. (2001) Survival with community-wide police-car deployment of automated external defibrillators in a large metropolitan area, Abstract. Pacing Clin Electrophysiol 24: 584

16. Groeneveld PW, Owens DK (2005) Cost-effectiveness of training unselected laypersons in cardiopulmonary resuscitation and defibrillation. Am J Med 118: 58-67

17. Hensel FJ (2002) Reanimation - Frühdefibrillation durch medizinische Laien. Dtsch Ärztebl 99: C361C362

18. Kern KB, Halperin HR, Field J (2001) New guidelines for cardiopulmonary resuscitation and emergency cardiac care: changes in the management of cardiac arrest. JAMA 285: 1267-1269

19. Larsen MP, Eisenberg MS, Cummins RO et al. (1993) Predicting survival from out-of-hospital cardiac arrest: a graphic model. Ann Emerg Med 22: 1652-1658

20. MacDonald RD, Morrley JL, Weinstein C (2002) Impact of prompt defibrillation on cardiac arrest at a major international airport. Prehosp Emerg Care 6: 1-5

21. Marenco JP, Wang PJ, Link MS et al. (2001) Improving survival from sudden cardiac arrest: the role of the automated external defibrillation. JAMA 285: 1193-2000

22. Nichol G, Laupacis A, Stiell IG et al. (1996) Cost-effectiveness analysis of potential improvements to emergency medical services for victims of out-ofhospital cardiac arrest. Ann Emerg Med 27: 711720

23. Nichol G, Hallstrom AP, Ornato IP et al. (1998) Potential cost effectiveness of public access defibrillation in the United States. Circulation 97: 13151320

24. NN (1998) Good samaritan civil immunity for use of automated external defibrillator. Tit. $42 \S$ 8331.2, PA, Consolidated Statutes

25. NN (2000) Guidelines 2000 for cardiopulmonary resuscitation and emergency cardiovascular care. 4. The automated external defibrillator: key link in the chain of survival. Circulation [Suppl 1] 102:60 I-76

26. NN (2001) Strafgesetzbuch (StGB) vom 15. Mai 1871 (RGBI S 127) in der Fassung der Bekanntmachung vom 13. November 1998 (BGBI I, 3322), zuletzt geändert durch das 34. Strafrechtsänderungsgesetz, §129b StGB (34. StrÄndG) vom 22. August 2002 (BGBI I, 3390), 26. neu bearb. Aufl. Beck, München
27. Page RL, Joglar JA, Kowal RC et al. (2000) Use of automated external defibrillators by a US airline. $\mathrm{N}$ Engl J Med 263: 1210-1216

28. Prior SG, Aliot E, Blomstron-Lundqvist $C$ et al. (2001) Task force on sudden cardiac death of the European Society of Cardiology. Eur Heart J 22: 1374-1450

29. Schäfer S, Pohl-Menthen U (Hrsg) (2001) Repräsentative Bevölkerungsbefragungen (1993 und 2000): Erste Hilfe-Kenntnisse in der Bevölkerung. Institut für den Rettungsdienst, Schriftenreihe zum Rettungswesen, Bd 25. Verlags- und Vertriebsgesellschaft DRK, Landesverband Westfalen-Lippe, Nottulm

30. Sefrin P (2001) Frühdefibrillation durch Ersthelfer Risiko oder Qualitätssprung? Notarzt 17: 90-92

31. Sefrin P, Rupp J (1979) Reanimation im Notarztwagen. Münchn Med Wochenschr 121: 1575-1578

32. Sefrin P, Schäfer R (1986) Reanimation durch Laien? Anästh Intensivther Notfallmed 21: 273-279

33. Swor R, Compton S (2004) Estimating cost-effectiveness of mass cardiopulmonary resuscitation training strategies to improve survival from cardiac arrest in private locations. Prehosp Emerg Care 8: 420-423

34. Trappe HJ, Andresen D, Arntz HR et al. (2005) Positionspapier zur „Automatisierten Externen Defibrillation". Z Kardiol 94: 287-295

35. Valenzuela TD, Roe DJ, Nichol Get al. (2000) Outcomes of rapid defibrillation by security officers after cardiac arrest in casinos. N Engl J Med 343: 12061209

36. Wassertheil J, Keane G, Fisher N et al. (2000) Cardiac arrest outcomes at the Melbourne cricket ground and shrine of remembrance using a tiered response strategy - a forerunner to public access defibrillation. Resuscitation 44: 92-104

37. Zipes DP (2001) Saving time saves lives. Circulation 104: 2506-2508

38. Zipes DP, Wellens JHH (1998) Sudden cardiac death. Circulation 98: 2334-2351 